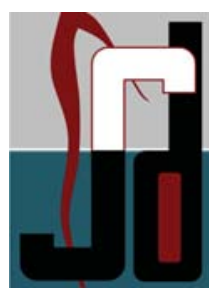

\title{
PICTURE PRESENTATION
}

\section{Endodontic Management with Mesiobuccal Root Resection of Bilateral Maxillary First Molar: A Picture Presentation}

Sathyanarayanan $\mathrm{R}^{1}$, Gayathri $\mathrm{N}^{2}$

ABSTRACT: Root resection or amputation is carried for isolated root damage because of caries or periodontal origin. The success of the treatment will depend on the careful treatment planning, successful endodontic therapy and precise post endodontic restoration. This picture presentation shows an unusual bilateral mesio buccal caries destruction with trifurcation involvement and the steps in managing such case.

Keywords: Root resection, endodontic therapy, post endodontic restoration, trifurcation

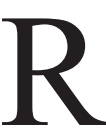

oot resection is the surgical procedure by which one or more of the roots of a multirooted tooth are removed at the level of the furcation whilst the crown and the remaining roots are left in function

The Indications can be either periodontal or endodontic origin ${ }^{[1-4]}$

Conventional periodontal indications include:

1. Moderate to advanced furcations involvement

2. Severe bone loss affecting one or more $\operatorname{root}(s)$

3. Severe recession or dehiscence of a root

4. Unfavourable root proximity between adjacent teeth .

Endodontic indications include ${ }^{[5]}$ :

1. Root fracture or perforation

2. External root resorption

3. Failed root canal treatment

\section{Root caries}

5. Endodontic- periodontal combined lesions

After careful selection of case, the root canal therapy is performed or if it is already done and in satisfactory condition, then root resection is done followed by satisfactory post endodontic restoration. Permanent crowns are given after complete soft tissue healing at earliest possible time. The success of this therapy depends on successful endodontic therapy and the longevity of the treatment depends on the good prosthesis work with excellent crown tooth margin ${ }^{[6]}$. This picture presentation demonstrates and rationalizes the
Scan the QR code with any smart phone scanner or PC scanner software to download/ share this publication

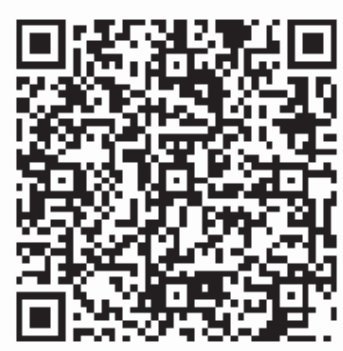


step by step procedure of management unusual bilateral caries involvement of mesio buccal root of maxillary first molar.

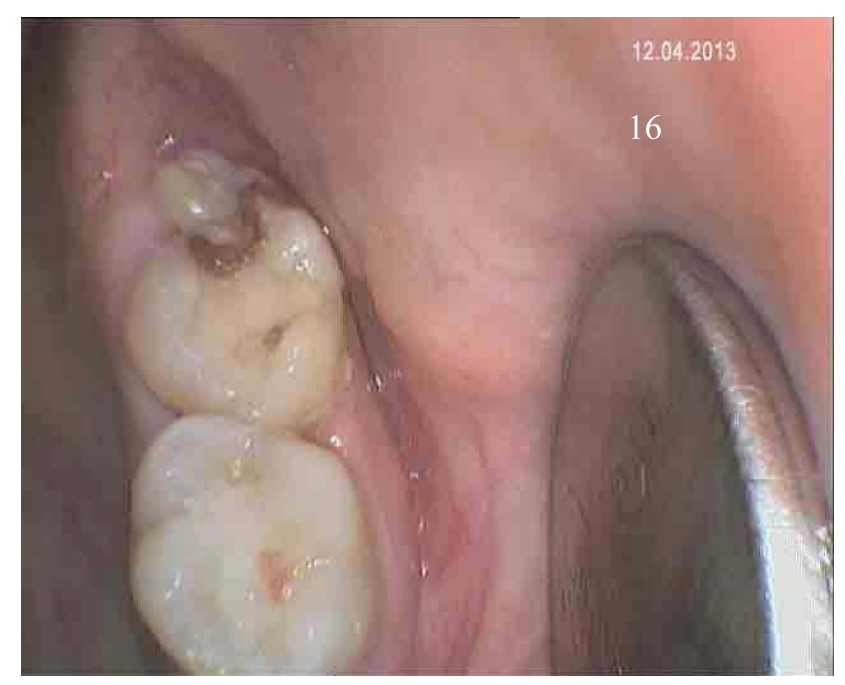

A 31 year old woman presented with a complaint of food impaction and occasional discomfort related to 16 and 26

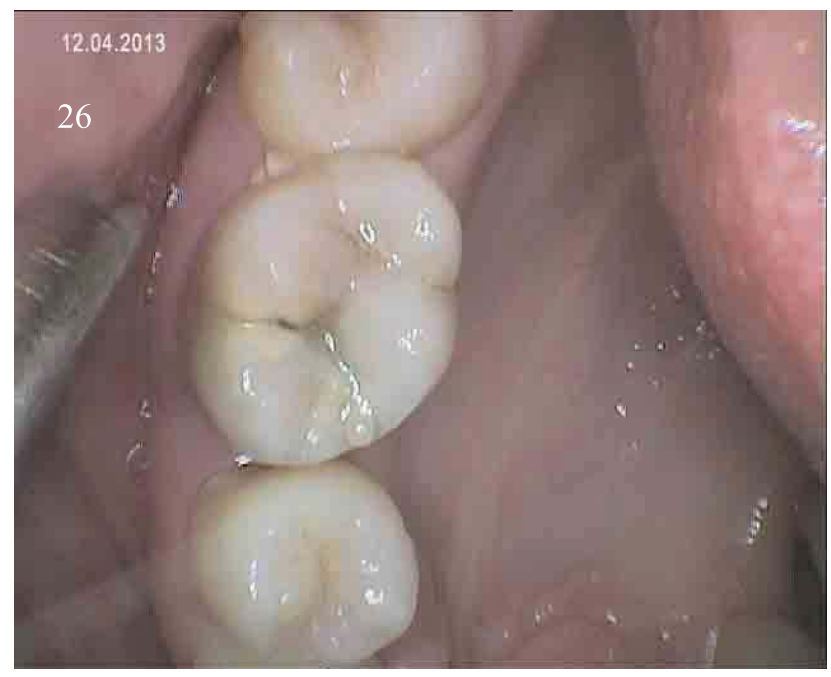

Fig1:Preoperative image of 16 and 26

Clinically, patient complain was food present in 26. On mesial aspect deep impaction for past 6 years and occasionally pain every few months. The pain episodes was brought under control by the analgesics. Occlusally, tooth colored restoration was periodontal pocket were present on 16 and 26. Pocket was more deep on 26 than 16. expect bleeding on probing on mesial aspect of both 16 and 26 present in 16 and pit and fissure caries was
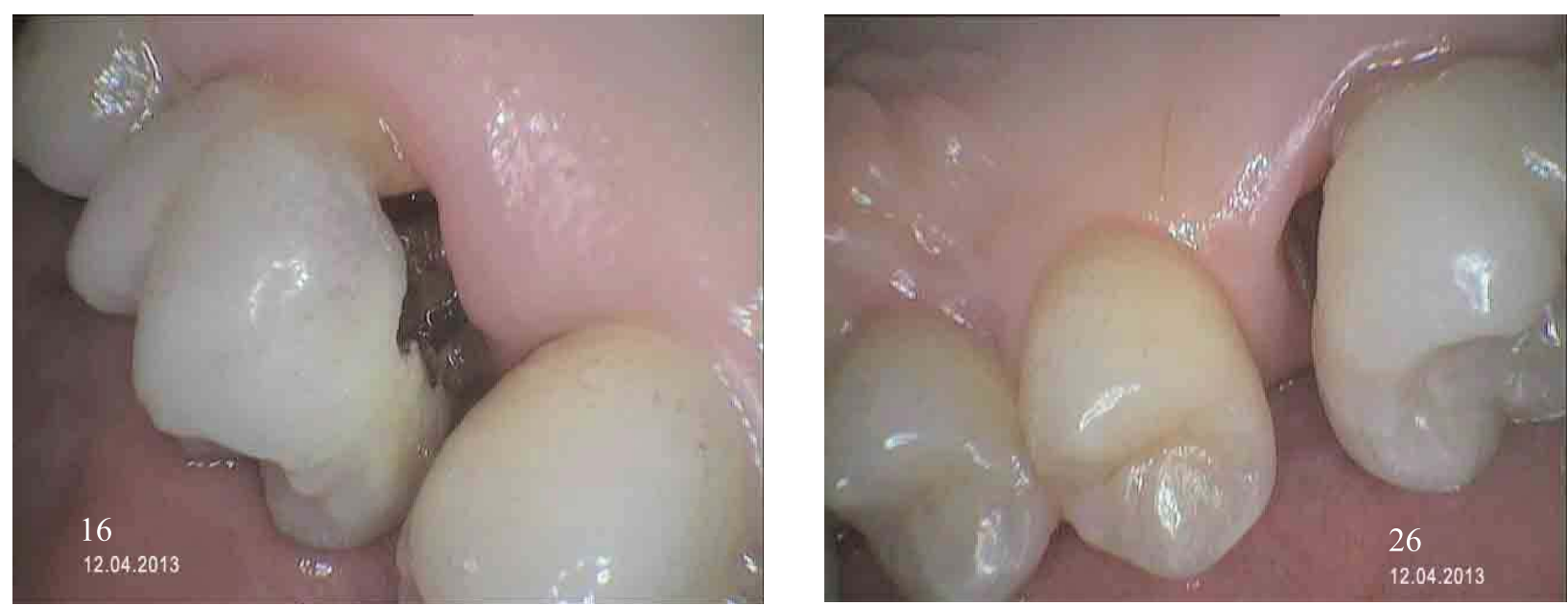

Fig 2: Preoperative close up image of 16 and 26

Diastema was present on mesial aspect of both 16 and 26 . When viewed from buccal aspect, burrowing caries lesion was seen in 16 and 26. The destruction was more gross in 16 than 26. As the diastema was present, creating positive contact with second premolar will require over contouring of the final crown and the remaining tooth structure should be sufficiently strong to provide additional support. Tooth exhibited no mobility and hence was decided to do further evaluation radiologically. 

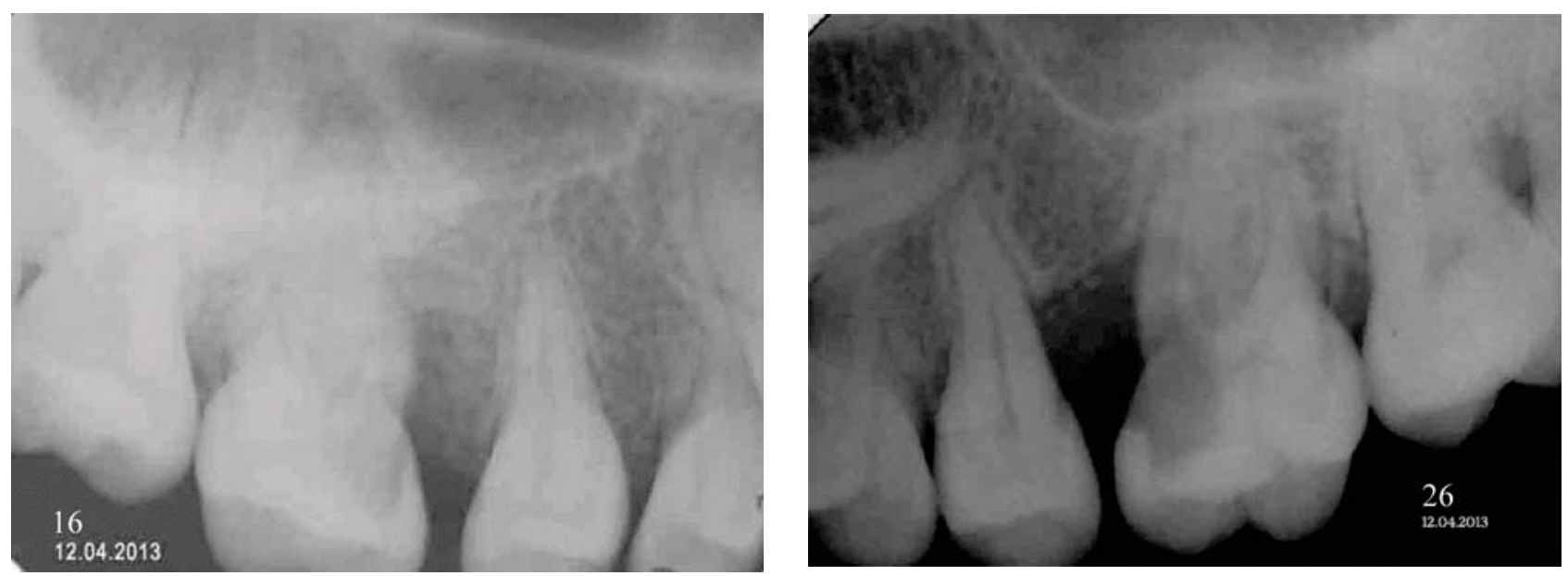

Fig 3: Preoperative radiograph of 16 and 26

Radiographically, 26 showed gross destruction than 16 , with bone loss extending up to middle third of the mesio buccal root. 16 radiograph showed periapical radiolucency in relation to palatal root and coronal radiolucency was not very evident radiographically. Summarizing, clinical and radiological finding, clinically 16 showed more structural damage, while radiologically

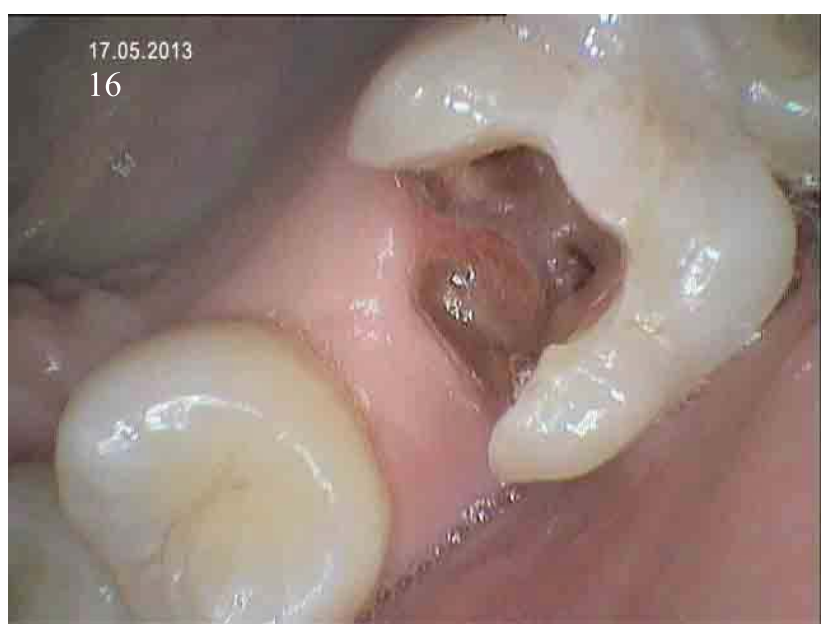

26 showed more damage. The canal were radiographically traceable in both the teeth. Hence it was assumed, root canal therapy may not be very difficult to carry out in both the teeth. The decision of treatment will be solely based on the extend of the structural damage and it was planned to excavate the caries lesion and reassess the damage for continuation of treatment.

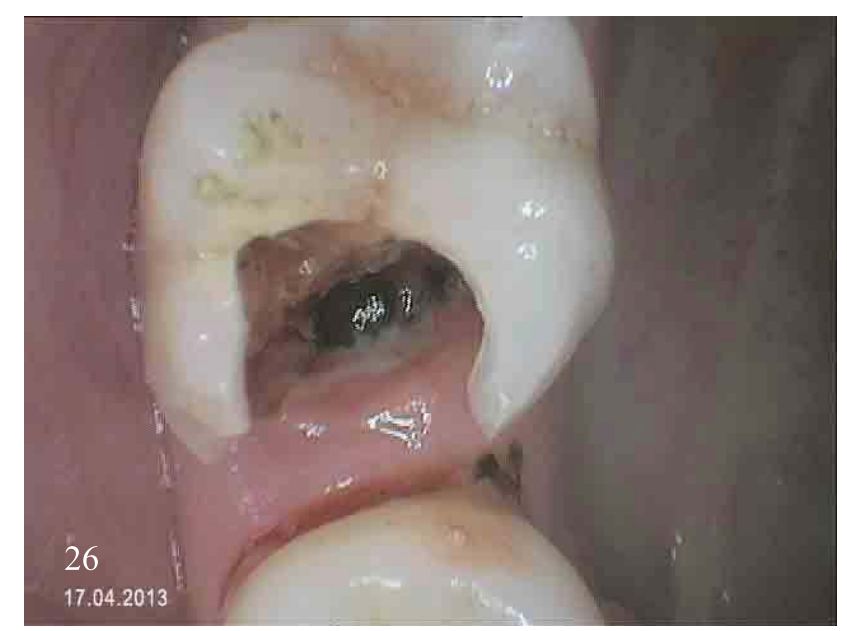

Fig 4: After caries excavation of 16 and 26

Caries excavation of 16 revealed trifurcation involvement with totally separated mesio buccal root Caries excavation of 26 revealed trifurcation involvement like 16 , but the mesio buccal root was still intact with the remaining tooth structure. At this juncture it was decided to proceed with root canal therapy, remove the mesio buccal root, reinforce the core material with radicular post and reevaluate for immediate crown or to postpone depending upon with healing of the tissue. 

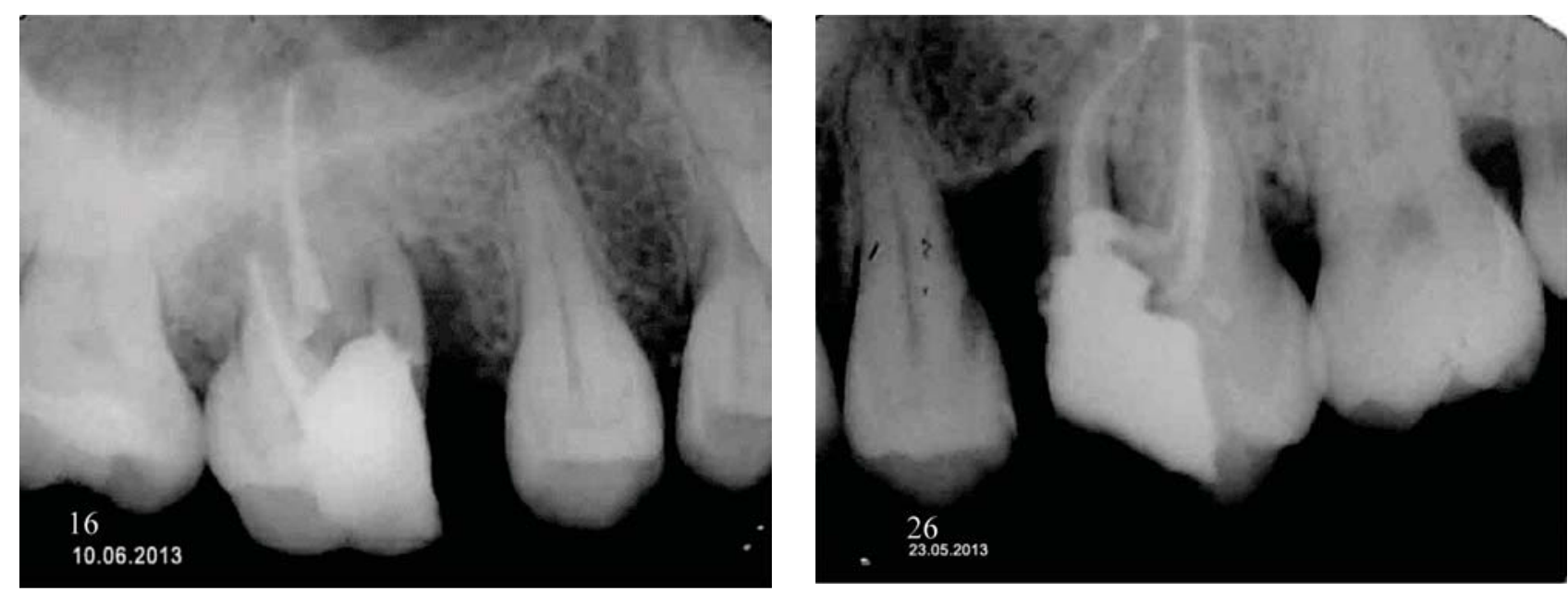

Fig 5: Post obturation radiograph of 16 and 26

Cleaning and shaping was done by crown down technique with protaper rotary system for both 16 and 26. Calcium hydroxideintracanal medicament was placed for two weeks and changed twice before obturation. No flare ups were present and the whole therapy was uneventful. Root canal were

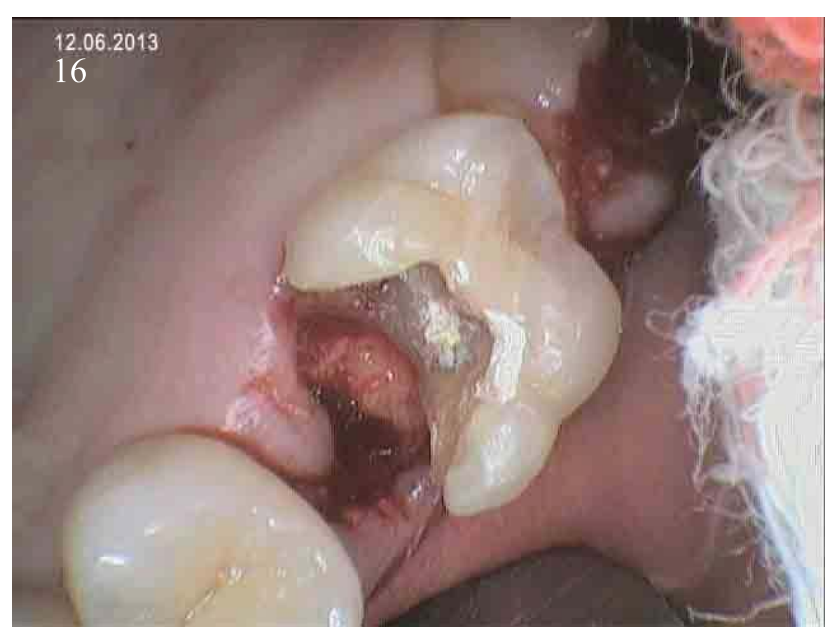

obturated with guttapercha points and zinc oxide eugenol sealer in lateral condensation technique. All the three canals were obturated in 26 and only palatal and distobuccal were obturated in 16. Post obturation revealed good obturation with adequate apical and lateral seal.

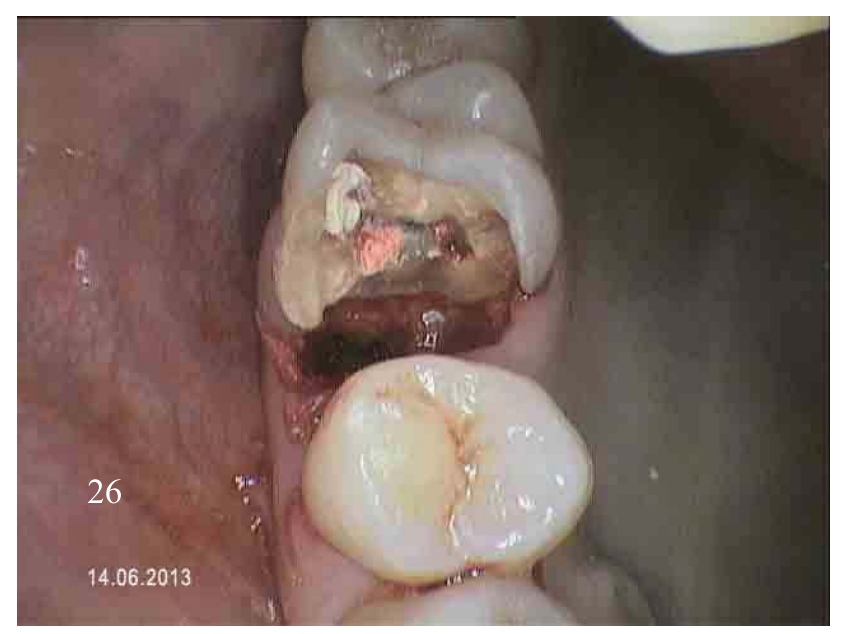

Fig 6: Mesio buccal root resection of 16 and 26

Mesio buccal root was separated with long shank tapering bur in 26. Both 16 and 26 mesio buccal root was elevated and removed with root elevators under local infiltration anesthesia. Haemostatis was acheived and the trifurcation was carefully examine under magnification for sharp margins and necessary contouring $^{[7,8]}$. The access cavity were restored with temoporary coronal seal. Post extraction instructions were given and mild analgesic was prescribed for three days. No antibiotic was given. 

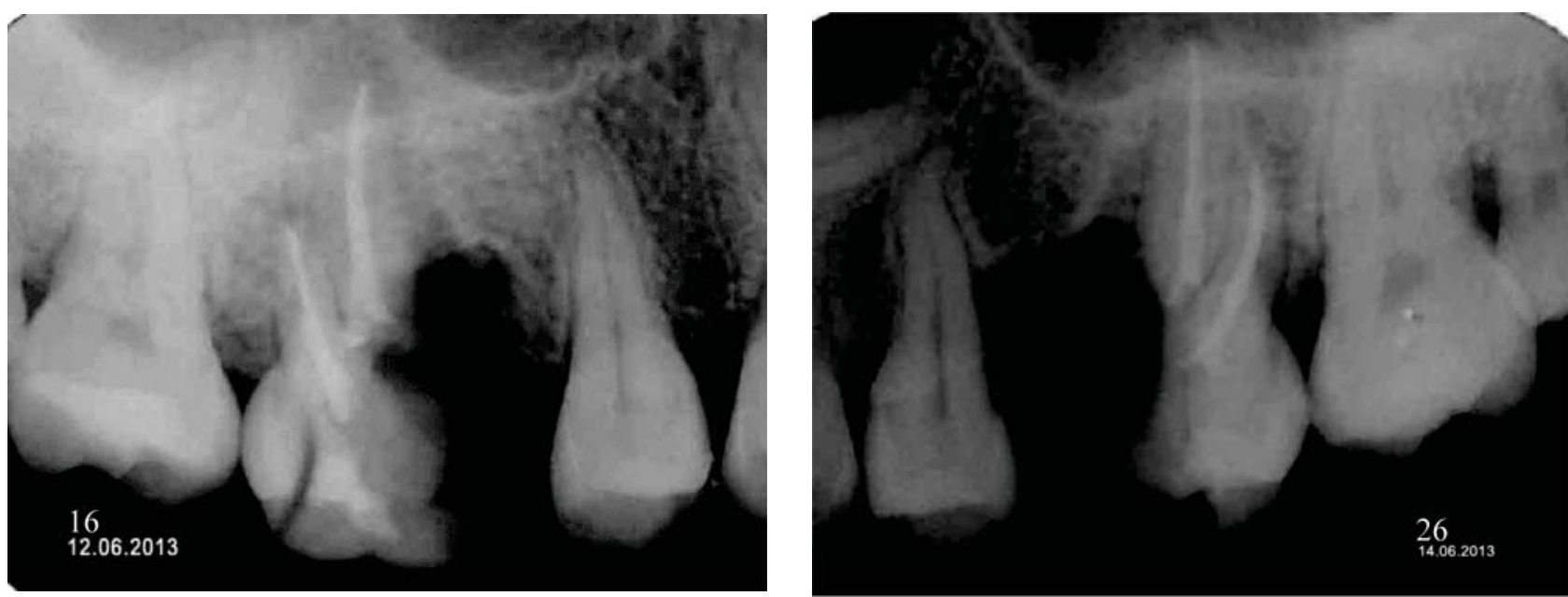

Fig7: Post root resection radiograph of 16 and 26

Post resection radiograph revealed satisfactory root resection. 16 showed more irregular
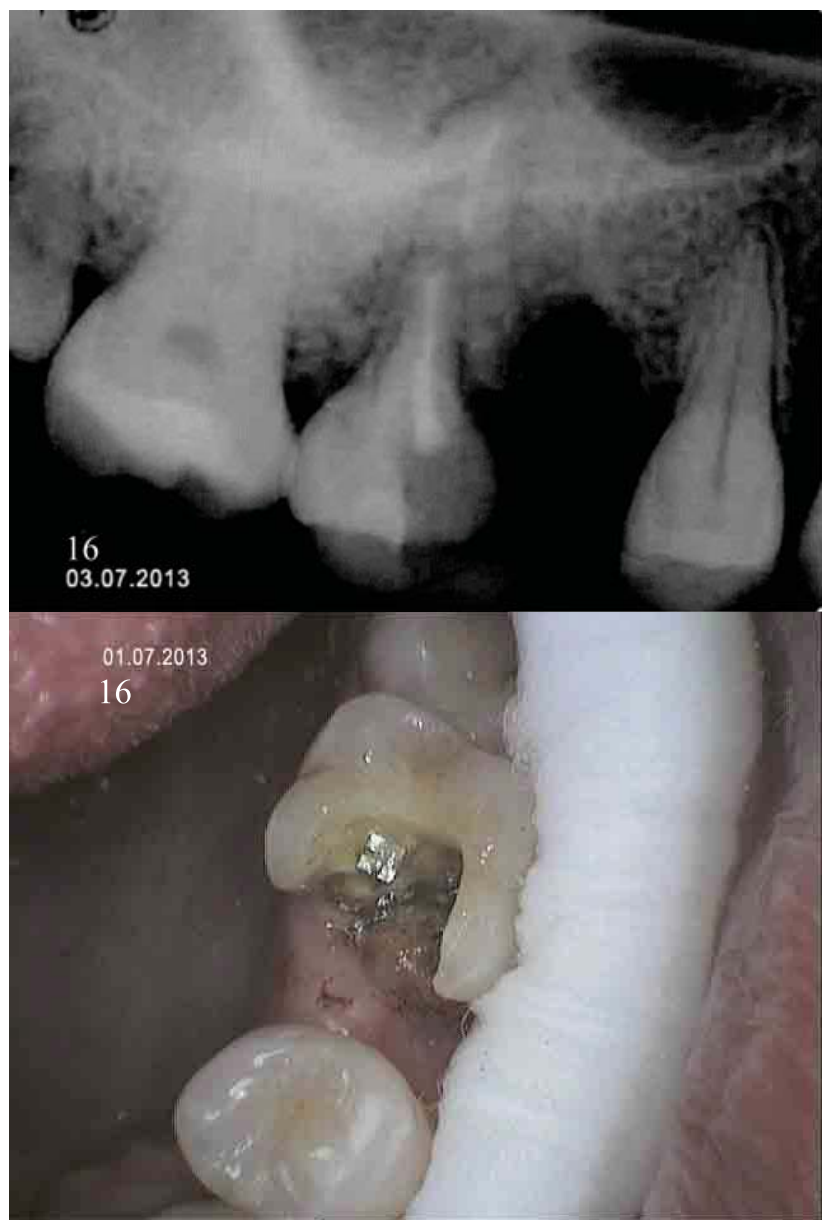

Fig 8: Post space radiograph of 16 \& Post selection radiograph of 26 Post selection photograph of 16 and 26

Guttapercha was removed from two third of palatal root canal with GG drill and post space were prepared with pesso drills. Pre fabricated metal post (Angelus, Brazil) were selected. margins and was predicated to have more difficulty in post and core build up and crown procedures.

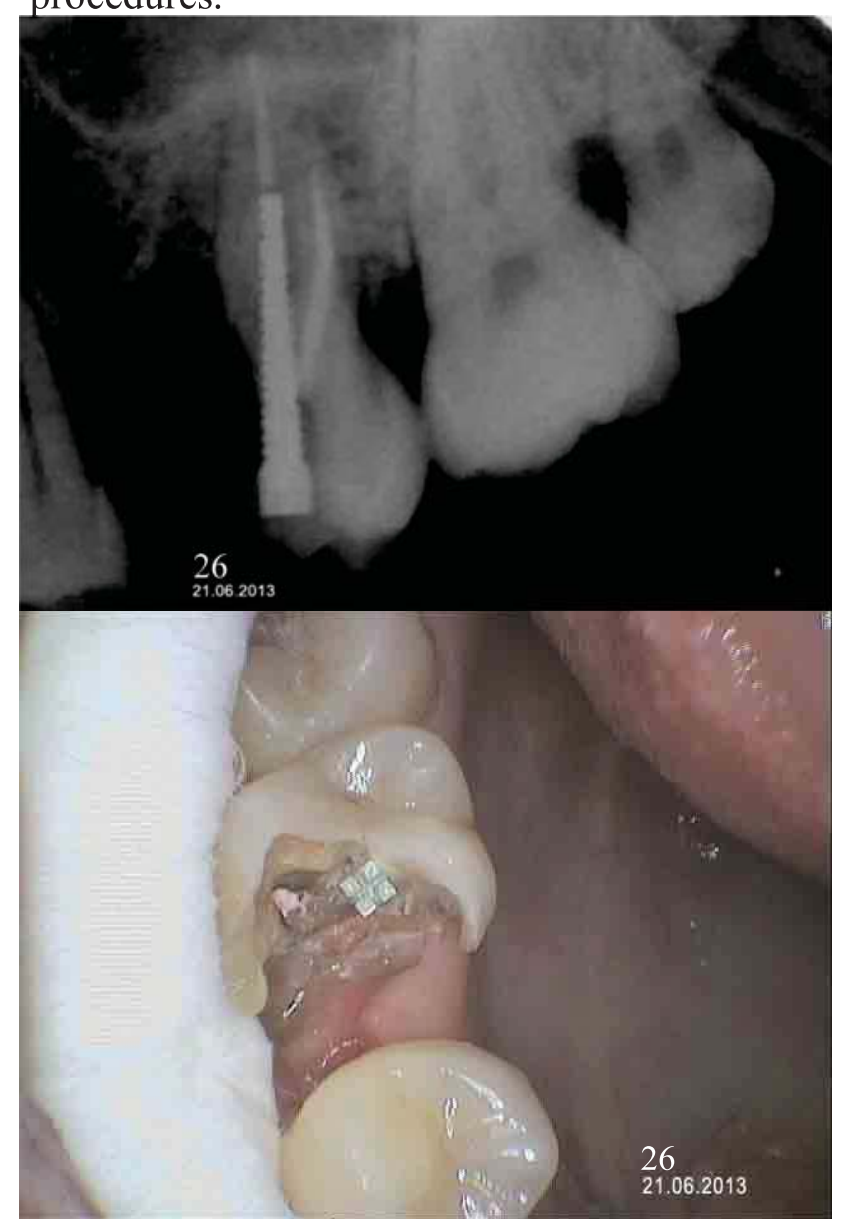

Metal post was selected over resin fibre post because of its rigidity and the difficulty in achieving moisture for root canal bonding procedures. Metal post were cemented with type I glass ionomer cement.(GC, Japan) 

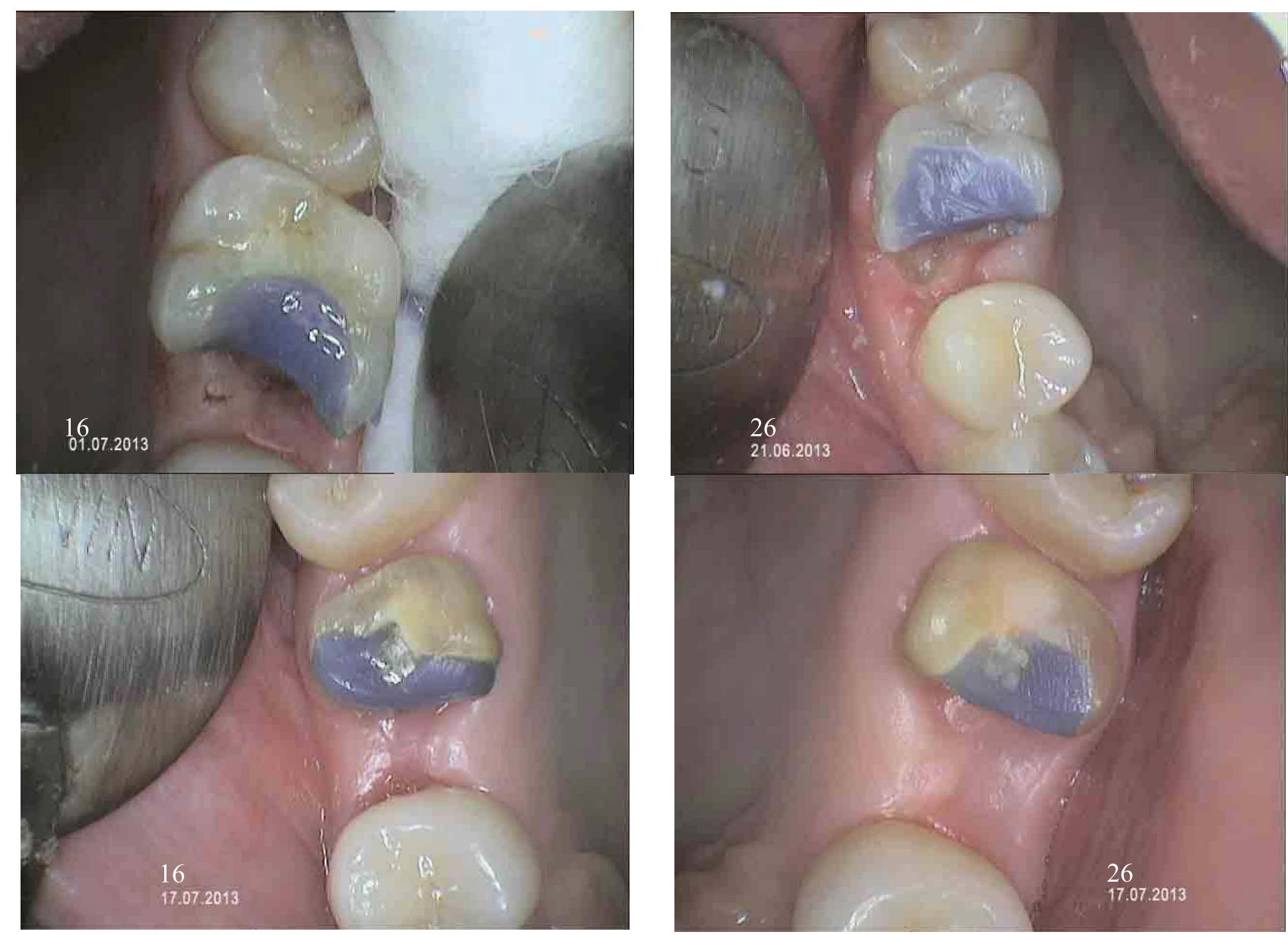

Fig 9: Core build up in 16 and 26

Composite resin core build up was done with flowable core matrial, Luxacore-Z (DMG,Germanny). Sixth generation bonding agent was used(Clearfil SE Bond). This is two bottle system, with self etching primer in one bottle and bonding in another bottle. Since it does not require any washing and drying, moisture control is more predictable. And

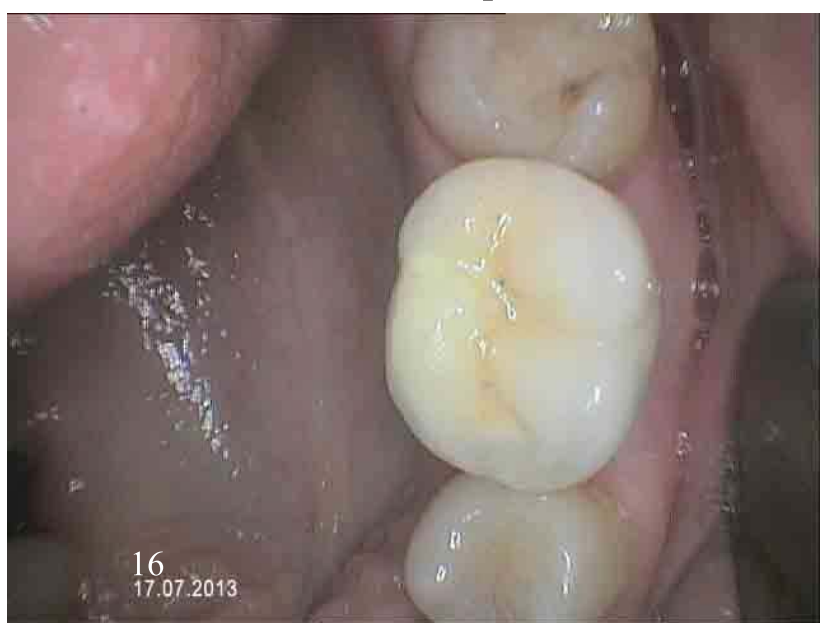

Luxacore $\mathrm{Z}$ can be used as cementing material and core material, thus giving advantage over packable core material. The contrast color is used as it easy to visualize the difficult margin and remove the excess material from the resected area. The second set of images shows excellent healing in two weeks

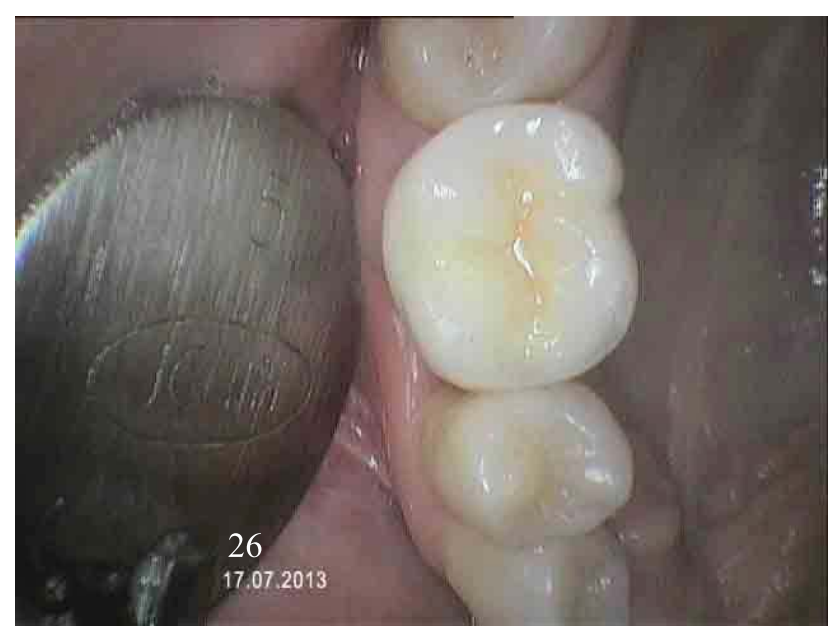

Fig 10: Occlusal view:After cementation of crown in 16 and 26 

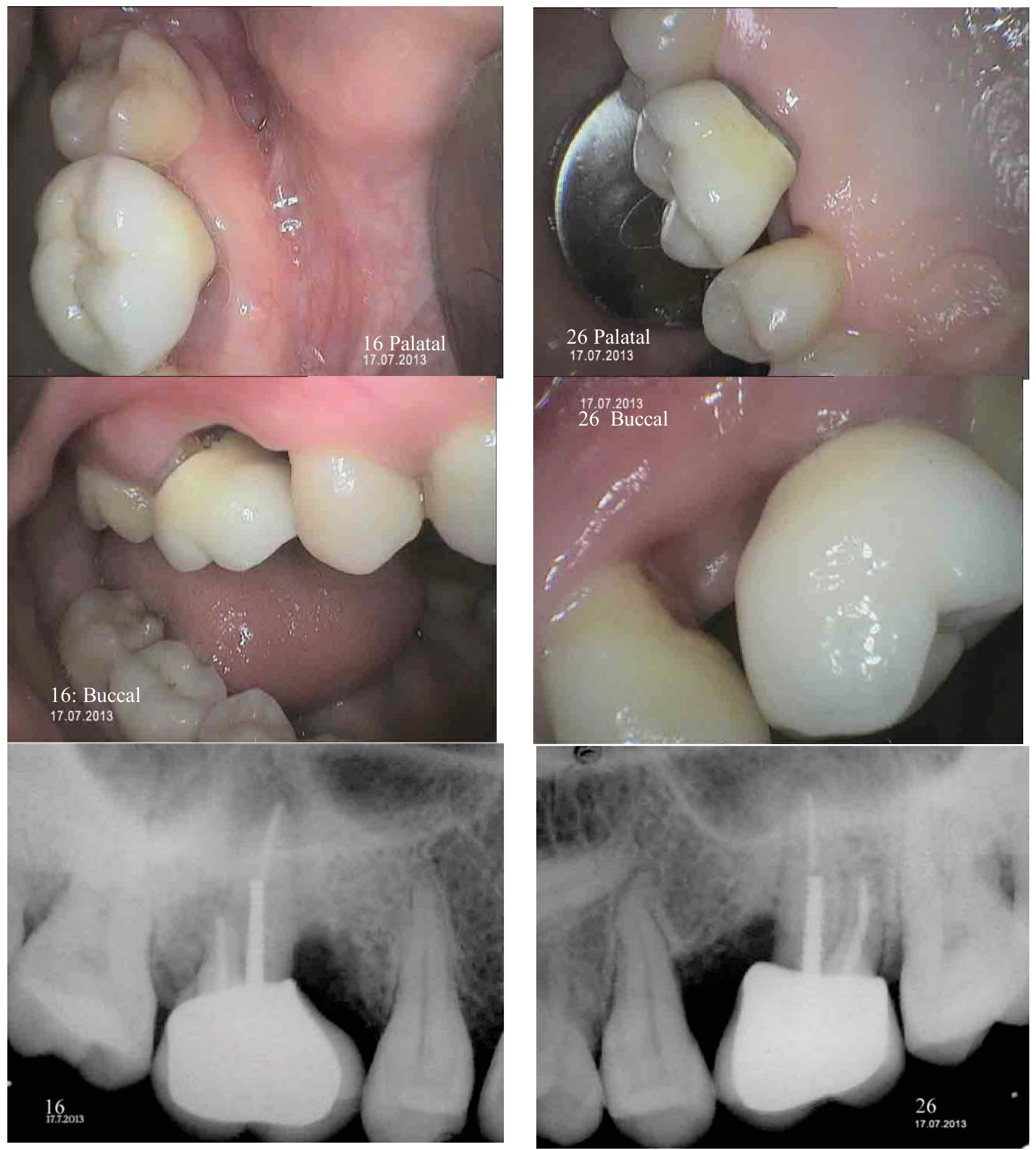

Fig 11: Post crown clinical image and radiograph

Post operative buccal and palatal view reveals very good marginal adaptation of both 16 and 26. The crown contour has been made so that it can be self cleansing thus able to maintain the health of periodontium. Post operative radiograph reveals excellent marginal adaptation of 16 on the mesial side and 26 crown margin shows over hang margin; however clinical examination/ image revealed the crown to be within acceptable limit.

\section{CONCLUSION:}

The following points are vital and can be summarized from this picture presentation:

- $\quad$ Roots should be well separated

- Minimum 1:1 of crown root ratio is mandatory 
- Metal cementable post is preferable

- Post should extend two thirds of root to provide adequate core support

- Flowable core maerial is preferable than packable

- $\quad$ Self etching bonding agent technique is

\section{REFERENCES:}

1. DeSanctis M, Murphy KG.. The role of resective periodontal surgery in the treatment of furcation defects. Periodontology.2000;22(feb):154-168

2. Yuh DY, Cheng GL, Chien WC, Chung CH, Lin FG, Shieh YS, Fang WH, Mau LP, Fu E, Huang RY.Factors affecting treatment decisions and outcomes of root-resected molars: a nationwide study.J Periodontol. 2013 Nov;84(11):1528-35.

3. Kasaj A.Root resective procedures vs implant therapy in the management of furcation-involved molars.Quintessence Int. 2014 Jun;45(6):521-9.

4. Oh SL.Mesiobuccal root resection in endodonticperiodontal combined lesions.IntEndod J. 2012 Jul;45 (7):660-9.

5. JohnsonTW,JostesLJ.Endodonticsurgery.In:JohnsonT W,Philadelphia,Color atlas of Endodontics :W.B Saunders company;2001

6. JohnsonRB,FayadIM,WitherspoonED.PeriradicularSur gery. In: CohenS, Missouri, Berman LH editors.

\section{Address for correspondence:}

Dr.Sathyanarayanan R

Department of Conservative Dentistry and Endodontics, Indira Gandhi Institute of Dental Sciences,

Sri Balaji Vidyapeeth University,

Pondicherry.

sathya@onlinedentallearning.com reliable with respect to moisture control.

The longevity of the treatment will depend on the proper case selection, careful endodontic, post endodontic, periodontic and prosthdontic protocols 9 .

Pathways of the pulp.10th Edition. Mosby Elseiver publishing;2011.

7. GeraldN,Glickman,GaryRH.EndodonticSurgery.In:Ing leIJ,BaklandKL,BaumgartnerJC,Ontario,BC Decker Inc.Ingle's Endodontics. 6thEdition.CBS Publishers and distributors;2013

8. BernabeFP,HollandR.EndodonticSurgery.In:EstrelaC, Brazil,EndodonticScience.Artes Medicas;2009.

9. Basten $\mathrm{CH}$, Ammons WF Jr, Persson R.Long-term evaluation of root-resected molars: a retrospective study.Int J Periodontics Restorative Dent. 1996 Jun;16 (3):206-19.

Authors:

${ }^{1}$ Professor and HOD, ${ }^{2}$ Reader, Department of Conservative Dentistry and Endodontics, Indira Gandhi Institute of Dental Sciences' Sri Balaji Vidyapeeth University Puducherry

How to cite this article:

Sathyanarayanan R, Gayathri N. Endodontic Management with Mesiobuccal Root Resection of Bilateral Maxillary First Molar: A Picture Presentation. Journal of Scientific Dentistry 2014;4(1):70-77

Source of Support: Nil, Conflict of Interest: None declared 